Martínez Juárez, M.; González Morga, N. y Pérez Cusó, J. (2018). Aproximación al perfil formativo del orientador profesional en la blogosfera. Revista de Investigación Educativa, 36(1), 39-56.

DOI: http://dx.doi.org/10.6018/rie.36.1.306401

\title{
Aproximación al perfil formativo del orientador profesional en la blogosfera
}

\author{
Approach to the carreer counselor's training \\ profile in the blogosphere
}

\author{
Mirian Martínez Juárez, Natalia González Morga y Javier Pérez Cusó \\ Dpto. de Métodos de Investigación y Diagnóstico en Educación. Universidad de Murcia
}

\section{Resumen}

La expansión de Internet y la web 2.0 en todos los escenarios sociales convierten a los blogs en una herramienta de difusión, comunicación y debate con múltiples potencialidades para el desarrollo y mejora del proceso orientador. Este trabajo analiza el perfil formativo del orientador en la blogosfera respondiendo a las cuestiones de quiénes son, qué ofrecen o qué necesidades formativas tienen. Se lleva a cabo a través de una investigación de diseño mixto que combina el análisis de contenido de 136 blogs representativos de la población con una entrevista estructurada, aplicada a 65 blogueros-participantes. Para el análisis de los datos se recurre a la estadística descriptiva e inferencial a través del programa estadístico SPSS v23 y al programa ATLAS.ti 7. Los resultados muestran una importante heterogeneidad en la formación inicial y complementaria de los blogueros, en su experiencia profesional y en los contenidos que publican; además destacan entre sus necesidades formativas la actualización constante de sus competencias técnicas para un mejor uso de los blogs, así como en nuevas tendencias en el ámbito de la orientación profesional. Estos resultados señalan la importancia de integrar las realidades off y on line en el proceso orientador a fin de conseguir una intervención más actual, eficaz y de mayor alcance.

Palabras clave: orientación profesional; internet; redes sociales (en línea); tecnologías web 2.0.

Correspondencia: Mirian Martínez Juárez, mmartinez@um.es. 


\begin{abstract}
The expansion of the Internet and web 2.0 in all social spheres makes blogs a tool of diffusion, communication and debate with many possibilities in the development and improvement of the guiding process. This paper analyzes the counselor's training profile in the blogosphere, answering questions about who they are, what they offer or what necessary professional development they have. This research combines content analysis of 136 blogs representative of the population and an interview applied to 65 bloggers. We use descriptive and inferential statistics and alternate the use of the statistical program SPSS version 23 and ATLAS.ti 7. The results show a significant heterogeneity in bloggers' initial and continuous training and in their professional experience and the content they publish. Among their training needs, the constant updating of technical competences and new trends in vocational guidance stands out. These results point to the importance of integrating online and offline realities in a guiding process in order to achieve a more effective, current and broader intervention.

Keywords: vocational guidance; internet; social networks (online); web 2.0 technologies.
\end{abstract}

\title{
Introducción
}

La Estrategia Europa 2020 (Comisión Europea, 2013), unida a la modernización del mercado laboral mediante el desarrollo de las competencias, fija distintas medidas dirigidas a estimular el crecimiento de la economía para el periodo 2014-2020, con actuaciones destinadas a potenciar el despliegue de Internet y las TIC. Nuestro país apoya este crecimiento tecnológico y actualmente el 80.6\% de la población entre 16 y 74 años utiliza este medio con frecuencia (Instituto Nacional de Estadística [INE], 2016).

La evolución de la tecnología promueve la denominada cuarta revolución industrial (Work Economic Fourm [WEF], 2016) y surgen nuevos retos y desafíos profesionales; se crea un nuevo escenario de relaciones multidireccionales, donde la generación y actualización continua de la información reformula la sociedad del aprendizaje. A corto plazo, se requerirán competencias desconocidas en nuevos contenidos, medios, métodos y formas de trabajo que exigirán un aprendizaje permanente que permita aplicar los conocimientos de forma innovadora en los puestos de trabajo.

Las exigencias de la población crecen, así como la diversidad de usuarios, por lo que la complejidad de la intervención orientadora aumenta. Es necesario adoptar una visión más amplia de la orientación, con la intención de desarrollarla a lo largo de la vida, para lo que será indispensable asumir estrategias, métodos y recursos más innovadores (Ceinos, 2009). Actualmente hay consenso en considerar que la inclusión de las TIC en la orientación permite aprovechar sus múltiples ventajas para favorecer la labor del orientador, facilitar una mayor aproximación a los distintos destinatarios e incrementar la calidad del proceso para hacerlo sostenible y funcional. La orientación profesional, ante esta sociedad compleja y dinámica, debe dar respuesta a estas nuevas necesidades y promover competencias como la flexibilidad, la adaptación, la apertura crítica, el respeto mutuo o la seguridad en uno mismo (Santana, 2013).

Actualmente, surge la e-orientación profesional, como un escenario de investigación relativamente reciente. Diferentes son las iniciativas que constatan la importancia de 
las TIC para llevar a cabo procesos de orientación eficaces (Ceinos, 2008; Fernández González, 2015; Muñoz-Carril y González-Sanmamed, 2014; Pantoja y Zwierewicz, 2008; Romero y Montilla, 2015; Santana y Santana, 2011; Sobrado, Ceinos y García, 2012).

Las TIC proporcionan múltiples posibilidades para el acceso, tratamiento y difusión de la información, rompen las barreras espacio-temporales y posibilitan una mayor motivación en los usuarios, para quienes cada vez es más atractivo el ciberespacio para satisfacer sus necesidades personales y profesionales. Estas potencialidades favorecen tanto la finalidad última de la orientación, como la flexibilidad, autonomía y personalización a través de una interacción basada en diferentes códigos y formas de comunicación que ofrecen respuestas más adaptadas a las características y demandas de los destinarios (Sobrado y Ceinos, 2011).

El desarrollo constante de la web 2.0, entendida como un espacio de intercambio dinámico de información y de interacción social, hace que todo esto sea posible en el ámbito que nos ocupa; entre las distintas herramientas que ofrece el entorno virtual se encuentran los blogs, un recurso óptimo para la expresión individual, la comunicación interpersonal y la construcción de una identidad o marca personal en el ciberespacio (Estalella, 2005).

En la blogosfera cualquier persona puede crear contenidos, expresar sus ideas y perspectivas sobre cualquier temática de manera sencilla y libre de costes (Jou, 2009). Esto puede justificar el incremento de profesionales de la orientación que optan por el uso de esta herramienta 2.0 para extender su dedicación laboral a la Red, a fin de compartir sus experiencias y orientaciones sin olvidar otras posibilidades como las de promocionar su actividad profesional, ampliar su red de contactos o aumentar su visibilidad (Barraza, 2013).

En los últimos años han proliferado los blogs relacionados con la orientación profesional, favoreciéndose así la posibilidad de desarrollar y poner en práctica procesos de orientación virtuales. La creación de estos espacios posibilitan al bloguero convertirse en un orientador en red, digital, 2.0 o en un e-orientador que guía o acompaña a los usuarios (lectores de su blog) a través de un proceso interactivo hacia su desarrollo profesional, creando así una comunidad virtual de orientación, sobre una estructura hipertextual, de inmediatez, actualización y comunicación bidireccional (Sáez Vacas, 2005).

El interés científico por estudiar la blogosfera se ha incrementado en los últimos años. A partir del año 2000 se presentan varios trabajos desde diferentes perspectivas y metodologías. Blood (2000) analiza el origen de los blogs; Fumero (2005) y Orihuela (2006) ofrecen una revisión teórica sobre su concepto, características y estructura, y Huertas (2010) se centra en sus tipologías. En el ámbito de la orientación es más frecuente encontrar el estudio de los blogs asociado a experiencias de innovación educativa (Aznar y Soto, 2010; Cuevas, 2011; Jou, 2009). En la búsqueda de antecedentes bibliográficos, se aprecia cierta tendencia hacia el estudio del uso de los blogs como herramienta innovadora para transformar una actividad profesional, mientras los trabajos centrados en las personas que lo gestionan son más bien escasos y pocas veces logran traducirse en trabajos empíricos con aportaciones de interés para la discusión (Flores, 2008). 
Algunos avances científicos sobre el perfil del bloguero en términos generales lo constituyen los trabajos de Bordignom (2007), de Ferreres y Garrido (2006) y Garrido y Lara (2008). En menor medida existen estudios que analizan el perfil del bloguero de áreas específicas como la orientación profesional, no siendo posible encontrar un estudio que sirva como referente directo en esta investigación. Reflexionar sobre el e-orientador profesional en la blogosfera es una forma de aproximarnos a una realidad sin explorar, pero necesitada de orden y coherencia para comprender y reconducir la intervención en el plano físico y virtual en la misma dirección. La continuidad y complementariedad en la práctica orientadora son claves para llegar a crear un sistema integrado de orientación actual, eficaz y de mayor alcance tanto para los usuarios como para lo profesionales de la orientación.

Los blogs plantean diversas oportunidades y retos a la praxis de la e-orientación, debido a la novedad de su introducción como recurso no restringido a acciones puntuales, así como a la necesidad de indagar en el perfil de los autores de aquellos blogs relacionados con esta temática.

El propósito del presente trabajo se centra en responder a cuestiones como quiénes son, qué ofrecen o qué necesidades tienen los blogueros que administran estos blogs de orientación profesional/laboral, lo que permitirá una aproximación al perfil del e-orientador que utiliza la blogosfera para difundir, informar, orientar, asesorar, formar... en relación a distintos contenidos.

\section{Método}

\section{Objetivos}

A partir del propósito planteado con anterioridad, los objetivos de este trabajo se concretan en:

- Conocer el perfil formativo de una muestra representativa de blogueros de orientación profesional a través de su formación inicial, complementaria y ocupación actual.

- Identificar los contenidos publicados en los blogs seleccionados y analizar su relación con distintas variables: tipología del blog y sexo, formación y ocupación de los blogueros.

- Detectar las necesidades formativas de los autores de los blogs a partir de su propia percepción.

\section{Muestra}

La muestra participante queda compuesta por un total de $136 \mathrm{blogs}$, de los que un $75 \%(n=102)$ son de autoría personal $(66.3 \%$ hombres y $33.7 \%$ mujeres $)$ frente a un $25 \%$ corporativos $(n=34)$. En la tabla 1 se muestra la edad de los blogueros y la antigüedad de los blogs, apreciando que la edad media de los blogueros es de 43.20 años, con antigüedades medias similares en el caso de los blogs pesonales frente a los corporativos. 
Tabla 1

Edad de los blogueros y antigüedad de los blogs seleccionados

\begin{tabular}{lccccc}
\hline & & Media & D.T. & Max & Min \\
\hline \multirow{2}{*}{ Personal } & Edad autor/a & 43.20 & 7.39 & 61 & 30 \\
& Antigüedad blog & 5.14 & 2.60 & 12 & 0 \\
\hline \multirow{2}{*}{ Corporativo } & Antigüedad blog & 4.35 & 2.29 & 10 & 0 \\
\hline
\end{tabular}

\section{Procedimiento}

Este trabajo se encuadra dentro de una investigación más amplia de enfoque y diseño mixto en la que se diferencian dos etapas. La primera de corte cuantitativo con un diseño metodológico no experimental, descriptivo y transversal, mientras que la segunda es de corte cualitativo, basada en la reducción, disposición y transformación de los datos.

En la primera etapa, desarrollada a lo largo del primer semestre de 2016, se siguen los pasos que conforman el análisis de contenido, técnica que consiste en ponderar los contenidos a través de la concreción de diferentes categorías y su posterior codificación (Berelson, citado en Hernández, Fernández-Collado y Baptista, 2007).

Para ello se establece la definición del universo a analizar y se extrae una muestra representativa. El universo lo constituyen los blogs relacionados con la orientación profesional/laboral, localizados a través de directorios y motores de búsqueda, como Technorati o Google Blog Search. También se utiliza el Blogroll, uno de los elementos de la estructura de un blog, que muestra enlaces a blogs recomendados. La búsqueda termina una vez alcanzado el punto de saturación de la información: cuando los blogs encontrados no muestran nuevos contenidos y servicios y el blogroll remite a otros ya registrados.

Una vez realizada la búsqueda $(\mathrm{N}=221)$ se procede a efectuar la selección, optando por dos criterios: destinatarios del blog (no dirigido en exclusiva al ámbito educativo formal o al de recursos humanos) y nivel de actividad (al menos un post en los últimos 30 días). De esta forma la muestra definitiva queda constituida por 136 blogs.

Seguidamente se pasa a identificar las unidades de análisis o concretar los elementos a explorar para dar respuesta a los objetivos del estudio. Para analizar el perfil formativo se valora tanto la información del autor contenida en su blog, como su perfil personal en la red LinkedIn, considerándose los siguientes aspectos: sexo, edad, formación inicial, formación complementaria, experiencia profesional y ocupación actual. Otra unidad de análisis identificada son los post publicados a lo largo del primer trimestre de 2016, que constituyen un total de 4516 entradas. A partir de estos se pueden extraer los contenidos abordados por cada uno de ellos, cumpliendo con el segundo objetivo. 
El último paso de esta técnica es categorizar la información recabada y representar las variables de investigación. Como resultado se obtienen distintas categorías y subcategorías para codificar la formación y ocupación de los blogueros, así como los contenidos que éstos abordan.

Una vez realizada la codificación, se obtienen los totales para cada una de las categorías y se lleva a cabo el análisis de datos cuantitativo a través del software SPSS v23 mediante estadísticos no paramétricos, valorando un nivel de significación $p \leq .05$.

En la segunda etapa, desarrollada en el segundo semestre de 2016, se pretende complementar la información recabada con la percepción de los autores de los blogs seleccionados, para lo que se envía una entrevista estructurada vía e-mail a aquellos blogueros que aceptan participar en la investigación. La guía de preguntas se divide en dos partes: la primera comprende los datos de identificación del autor y del blog; la segunda se compone de 8 cuestiones abiertas relacionadas con las fortalezas y debilidades del mismo y con las necesidades formativas para su desarrollo, pregunta en la que se centra este trabajo.

El porcentaje de respuesta de los blogueros fue del $47.8 \%$, es decir, responden a la entrevista un total de 65. El análisis de datos cualitativo se lleva a cabo a través del programa ATLAS.ti 7.

\section{Resultados}

Como primer objetivo de este trabajo se plantea conocer la formación inicial y complementaria, así como la ocupación actual de los participantes, para lo que a continuación se analizan las variables relacionadas con formacion y ocupación.

En relación a la formación, debido a la alta dispersión de titulaciones, se procede a agrupar la formación de base en torno a 6 categorías: Administración y Dirección de Empresas (ADE); Derecho y Ciencias del Trabajo; Pedagogía, Psicología y Educación; Sociología, Filosofía y Publicidad, titulaciones técnicas (Matemáticas e ingenierías), y una última categoría referida a blogueros sin estudios universitarios. En la tabla 2 se puede apreciar el porcentaje de blogueros dentro de cada categoría, así como su formación complementaria.

Se evidencia que el grupo más numeroso es el de Pedagogía, Psicología o titulaciones afines (30.11\%), seguido de quienes estudiaron titulaciones de corte jurídico/ laboral (34.73\%). La formación complementaria se divide en las dos opciones más recurrentes: Gestión y Dirección de Recursos Humanos y Coaching. La mayoría de los blogueros opta por una formación complementaria relacionada con recursos humanos, excepto los de titulaciones técnicas. Destaca el hecho de que son las personas que realizaron estudios dentro de las Ciencias Sociales, pero más alejados del ámbito de la educación y, por tanto, de la orientación (Sociología, Filosofía y Publicidad), quienes han optado en mayor medida por complementar sus estudios con formación en recursos humanos (50\%) y Coaching (37.5\%). La prueba chi cuadrado no muestra diferencias significativas en la titulación según sexo, ni en relación a la formación inicial ni de postgrado. 
Tabla 2

Formación inicial y complementaria de los autores de los blogs seleccionados

\begin{tabular}{lcccc}
\hline Categorías & $\mathbf{n}$ & $\mathbf{\%}$ & $\begin{array}{c}\text { Coaching } \\
\mathbf{( \% )}\end{array}$ & $\begin{array}{c}\text { RRHH } \\
\mathbf{( \% )}\end{array}$ \\
\hline ADE & 17 & 18.28 & 5.88 & 5.88 \\
Derecho y Ciencias del Trabajo & 23 & 24.73 & 21.74 & 39.13 \\
Pedagogía, Psicología y Educación & 28 & 30.11 & 14.29 & 32.14 \\
Sociología, Filosofía, Publicidad & 8 & 8.60 & 37.50 & 50.00 \\
Titulaciones técnicas (Matemáticas, & 15 & 16.13 & 26.67 & 13.33 \\
ingenierías) & 2 & 2.15 & 0.00 & 0.00 \\
Sin estudios universitarios & & & & \\
\hline
\end{tabular}

Paralelamente, en la tabla 3 se puede apreciar la ocupación de los blogueros en torno a cinco categorías. El grupo más significativo es el de orientadores laborales (32.65\%), seguido de los profesionales de recursos humanos (29.59\%) y formadores o docentes $(14.29 \%)$. Puede apreciarse también que la participación de las mujeres en todas las categorías es inferior a la de los hombres, destacando especialmente en el grupo de formadores y docentes que baja hasta el 21.43\%. Por el contrario, el grupo profesional con una mayor representación femenina es el de orientadoras laborales $(39.39 \%)$.

Tabla 3

Ocupación actual de los autores de los blogs seleccionados

\begin{tabular}{|c|c|c|c|c|c|c|}
\hline \multirow[t]{2}{*}{ Ocupación } & \multirow[t]{2}{*}{$\mathbf{N}$} & \multirow[t]{2}{*}{$\%$} & \multicolumn{2}{|c|}{$\begin{array}{l}\text { Frecuencia de } \\
\text { publicación }\end{array}$} & \multirow{2}{*}{$\begin{array}{c}\text { Hombres } \\
(\%)\end{array}$} & \multirow{2}{*}{$\begin{array}{c}\text { Mujeres } \\
(\%)\end{array}$} \\
\hline & & & Media & D.T. & & \\
\hline Directores y gerentes & 12 & 12.24 & 3.60 & 7.69 & 64.29 & 35.71 \\
\hline Consultor / Técnico RRHH & 29 & 29.59 & 2.92 & 3.89 & 68.97 & 31.03 \\
\hline Formador / Docente & 14 & 14.29 & 2.02 & 1.40 & 78.57 & 21.43 \\
\hline Orientador laboral & 32 & 32.65 & 20.21 & 58.62 & 60.61 & 39.39 \\
\hline Otros & 11 & 11.22 & 8.76 & 14.52 & 63.64 & 36.36 \\
\hline
\end{tabular}

La tabla 3 también muestra la frecuencia media de publicación, el estadístico H de Kruskal-Wallis señala que las diferencias entre los distintos perfiles de ocupación son significativas (Chi-cuadrado=9.593, gl=4, sig.=.048), siendo los orientadores quienes publican más a menudo.

Para dar respuesta al segundo objetivo (Identificar los contenidos publicados en los blogs seleccionados y analizar su relación con distintas variables), se analizan los posts publicados, elaborándose un sistema de categorías excluyente que aparece en la tabla 4, ordenadas según sus totales. 
Tabla 4

Categorías de clasificación de los contenidos de los blogs seleccionados

Ofertas de empleo - Privadas. Difusión de ofertas o bolsas de empleo de em-
presas privadas.
Mercado laboral. Situación del mercado de trabajo, datos económicos, perfiles
profesionales demandados, yacimientos de empleo.

N $\%$

Competencias transversales. Conocimientos, habilidades y actitudes de corte personal y social potenciadores de la empleabilidad.

Ofertas de empleo - Público. Difusión de ofertas o bolsas de empleo público.

Búsqueda Activa de Empleo. Proceso y técnicas de búsqueda de empleo

Emprendimiento. Información para el autoempleo (ayudas, formación, ideas de negocio).

Formación - Oferta. Información sobre oferta de acciones formativas.

Gestión de RRHH. Contenidos dirigidos a profesionales de este ámbito.

Eventos. Referencias a congresos, jornadas, ferias... de empleo y/u orientación.

Derecho laboral. Información legal y derechos de los trabajadores.

Off topic. Contenidos no relacionados con la orientación profesional.

Trabajo en el extranjero. Reflexiones, consejos e información para la búsqueda de empleo en el extranjero

Becas - Formación. Información sobre ayudas para la realización de estudios.

Marca personal. Contenidos dirigidos a la creación de una identidad profesional.

Formación - Reflexiones. Análisis y discusión en torno a la formación.

Becas - Prácticas. Información sobre ayudas para la realización de prácticas.

Redes sociales. Información y reflexiones en torno a la utilización de las redes sociales tanto en el plano profesional como personal.

Desarrollo de la carrera profesional. Clarificación y establecimiento de metas y objetivos profesionales, así como el proceso a seguir para su concreción y consecución.

Coaching. Contenidos relativos a procesos de entrenamiento para el desarrollo de determinadas competencias o mejora en la realización de distintos procesos.

Meta-blog. Contenidos relativos al propio blog: evolución, personas invitadas, participaciones esporádicas, etc.

Becas - Otros. Información sobre ayudas para la investigación o de voluntariado.

Cualificación y acreditación. Certificados de profesionalidad y acreditación de competencias.

Orientador. La figura del orientador: perfil, desarrollo y formación. 
La categoría con mayor presencia es la relativa a ofertas de empleo privado $(15.40 \%)$, seguida de mercado laboral $(12.87 \%)$, competencias transversales $(10.66 \%)$ y ofertas de empleo público (9.97\%). Por el contrario, la categoría con menos entradas es la de orientador, con un $0.22 \%$.

Más allá de conocer los contenidos con mayor presencia en la blogosfera es interesante conocer la relación de algunas variables de los propios blogueros con los contenidos que publican, por lo que a continuación se presentan los análisis comparados en relación a la tipología del blog y el sexo, formación y ocupación de los autores de los blogs.

En cuanto al carácter personal o corporativo de los blogs, se realiza un análisis comparado a través de la prueba U de Mann-Whitney, como se muestra en la tabla 5. Para ello se compara el peso relativo de cada categoría de contenido con el total de publicaciones del blog.

Tabla 5

Contenidos publicados según tipología del blog. Prueba U de Mann-Whitney

\begin{tabular}{|c|c|c|c|c|c|c|c|}
\hline Categoría & $\begin{array}{l}\text { Tipología } \\
\text { blog }\end{array}$ & $\mathbf{N}$ & Media & D.T. & $\begin{array}{c}\text { Rango } \\
\text { promedio }\end{array}$ & $\begin{array}{l}\text { Suma de } \\
\text { rangos }\end{array}$ & $\begin{array}{l}\text { U de Mann- } \\
\text { Whitney } \\
\text { (Sig. ) }\end{array}$ \\
\hline \multirow{2}{*}{$\begin{array}{c}\text { Becas } \\
\text { (Formación) }\end{array}$} & Personal & 96 & .32 & 1.553 & 62.38 & 5988.50 & \multirow{2}{*}{$\begin{array}{c}1332.500 \\
(.021)\end{array}$} \\
\hline & Institucional & 32 & .81 & 2.086 & 70.86 & 2267.50 & \\
\hline \multirow{2}{*}{$\begin{array}{l}\text { Derecho } \\
\text { laboral }\end{array}$} & Personal & 96 & 1.28 & 4.750 & 61.94 & 5946.00 & \multirow{2}{*}{$\begin{array}{c}1290.000 \\
(.022)\end{array}$} \\
\hline & Institucional & 32 & 6.38 & 16.423 & 72.19 & 2310.00 & \\
\hline \multirow{2}{*}{$\begin{array}{l}\text { Mercado } \\
\text { laboral }\end{array}$} & Personal & 96 & 6.97 & 18.166 & 60.93 & 5849.00 & \multirow{2}{*}{$\begin{array}{c}1193.000 \\
(.023)\end{array}$} \\
\hline & Institucional & 32 & 9.41 & 16.584 & 75.22 & 2407.00 & \\
\hline \multirow{2}{*}{$\begin{array}{l}\text { Formación } \\
\text { (Ofertas) }\end{array}$} & Personal & 96 & 3.16 & 12.413 & 61.52 & 5906.00 & \multirow{2}{*}{$\begin{array}{c}1250.000 \\
(.025)\end{array}$} \\
\hline & Institucional & 32 & 6.34 & 13.743 & 73.44 & 2350.00 & \\
\hline \multirow{2}{*}{$\begin{array}{c}\text { Formación } \\
\text { (Reflexiones) }\end{array}$} & Personal & 96 & 2.36 & 9.301 & 60.74 & 5831.00 & \multirow{2}{*}{$\begin{array}{c}1175.000 \\
(.004)\end{array}$} \\
\hline & Institucional & 32 & 5.19 & 11.197 & 75.78 & 2425.00 & \\
\hline \multirow{2}{*}{$\begin{array}{l}\text { Ofertas de } \\
\text { empleo } \\
\text { privado }\end{array}$} & Personal & 96 & 2.08 & 8.332 & 61.49 & 5903.50 & \multirow{2}{*}{$\begin{array}{c}1247.500 \\
(.010)\end{array}$} \\
\hline & Institucional & 32 & 10.38 & 23.048 & 73.52 & 2352.50 & \\
\hline \multirow{2}{*}{$\begin{array}{l}\text { Ofertas de } \\
\text { empleo } \\
\text { público }\end{array}$} & Personal & 96 & 2.29 & 8.463 & 61.89 & 5941.50 & \multirow{2}{*}{$\begin{array}{c}1285.500 \\
(.026)\end{array}$} \\
\hline & Institucional & 32 & 2.59 & 6.579 & 72.33 & 2314.50 & \\
\hline \multirow{2}{*}{$\begin{array}{l}\text { Emprendi- } \\
\text { miento }\end{array}$} & Personal & 96 & 2.48 & 7.698 & 61.53 & 5907.00 & \multirow{2}{*}{$\begin{array}{c}1251.000 \\
(.032)\end{array}$} \\
\hline & Institucional & 32 & 5.31 & 13.292 & 73.41 & 2349.00 & \\
\hline \multirow{2}{*}{$\begin{array}{c}\text { Trabajo en el } \\
\text { extranjero }\end{array}$} & Personal & 96 & 1.19 & 5.046 & 62.25 & 5976.00 & \multirow{2}{*}{$\begin{array}{c}1320.000 \\
(.049)\end{array}$} \\
\hline & Institucional & 32 & 1.22 & 2.296 & 71.25 & 2280.00 & \\
\hline
\end{tabular}


Como puede comprobarse en la tabla 5, existen diferencias significativas en nueve categorías, siendo los blogs corporativos los que muestran un promedio de publicación mayor que los personales.

En la comparación por sexo, la prueba U de Mann-Whitney no arroja diferencias significativas $(p \leq .05)$ en ninguna de las categorías.

En cuanto a la tasa relativa de publicaciones según la formación de base del bloguero, sorprende encontrar (tabla 6) diferencias significativas tan sólo en la categoría relativa a competencias transversales. Puede apreciarse que son los blogueros con titulaciones más alejadas de las ciencias sociales y del comportamiento quienes obtienen mayores tasas de publicación (sin estudios universitarios, titulaciones técnicas y ADE).

En relación a la formación complementaria, la prueba U de Mann-Whitney no muestra diferencias significativas $(p \geq .05)$ en el tipo de contenidos que publican, a excepción de aquellos que tienen formación complementaria en Coaching que sí publican más contenidos relacionados con esta temática (Mann-Whitney=491.500, Sig.=.002).

Tabla 6

Diferencias significativas de publicación en la categoría Competencias Transversales según formación inicial. Prueba Kruskal-Wallis

\begin{tabular}{lcccc}
\hline & & \multicolumn{3}{c}{ Competencias transversales } \\
\cline { 3 - 5 } Titulación & N & $\begin{array}{c}\text { rango } \\
\text { promedio }\end{array}$ & Media & D.T. \\
\hline ADE & 16 & 56.09 & 39.44 & 28.258 \\
Derecho y Ciencias del Trabajo & 23 & 36.43 & 16.61 & 26.917 \\
Pedagogía, Psicología y Educación & 25 & 33.46 & 13.24 & 21.386 \\
Sociología, Filosofía, Publicidad & 7 & 40.50 & 18.71 & 30.198 \\
Titulaciones técnicas (Matemáti- & 15 & 61.47 & 50.53 & 39.031 \\
cas, ingenierías) & 2 & 69.25 & 57.50 & 27.577 \\
Sin estudios universitarios & & & $20.448(5) .001$ & \\
\hline \multicolumn{1}{c}{ Chi-cuadrado (gl) Sig. } & & & &
\end{tabular}

Para una mayor profundidad, en la tabla 7 se compara el porcentaje promedio de publicaciones según la ocupación del bloguero, encontrando diferencias significativas $(p \leq .05)$ en cuatro categorías. En tres de esas categorías (ofertas de empleo públicas, búsqueda activa de empleo y trabajo en el extranjero) son los orientadores quienes muestran una tasa superior, mientras que en la categoría relacionada con la publicación de ofertas de empleo privadas son tanto los orientadores como los encuadrados en la categoría otros (abarca diferentes ocupaciones alejadas de las contempladas, por ejemplo, periodista o decorador), quienes muestran una media de publicación mayor al resto. 
Tabla 7

Contenidos publicados según la ocupación del bloguero. Prueba de Kruskal-Wallis

\begin{tabular}{|c|c|c|c|c|c|c|c|}
\hline Categoría & & $\begin{array}{l}\text { Directores } \\
\text { y gerentes }\end{array}$ & $\begin{array}{l}\text { Consultor } \\
\text { / Técnico } \\
\text { RRHH }\end{array}$ & $\begin{array}{l}\text { Formador/ } \\
\text { Docente }\end{array}$ & Orientador & Otros & $\begin{array}{c}\text { Chi- } \\
\text { cuadrado } \\
\text { (Sig.) }\end{array}$ \\
\hline \multirow{3}{*}{$\begin{array}{l}\text { Ofertas } \\
\text { empleo } \\
\text { privadas }\end{array}$} & $\begin{array}{c}\text { Rango } \\
\text { promedio }\end{array}$ & 46.00 & 47.60 & 46.00 & 55.50 & 57.77 & \multirow{3}{*}{$\begin{array}{c}9.716 \\
.045\end{array}$} \\
\hline & Media & .00 & .30 & .00 & 4.69 & 6.54 & \\
\hline & D.T. & .000 & 1.643 & .000 & 11.880 & 17.275 & \\
\hline \multirow{3}{*}{$\begin{array}{l}\text { Ofertas } \\
\text { empleo } \\
\text { públicas }\end{array}$} & $\begin{array}{c}\text { Rango } \\
\text { promedio }\end{array}$ & 46.00 & 46.00 & 46.00 & 57.25 & 57.15 & \multirow{3}{*}{$\begin{array}{c}13.627 \\
.009\end{array}$} \\
\hline & Media & .00 & .00 & .00 & 5.88 & 2.08 & \\
\hline & D.T. & .000 & .000 & .000 & 13.640 & 4.873 & \\
\hline \multirow{3}{*}{$\begin{array}{l}\text { Búsqueda } \\
\text { Activa de } \\
\text { Empleo }\end{array}$} & $\begin{array}{l}\text { Rango } \\
\text { promedio }\end{array}$ & 34.88 & 47.90 & 43.19 & 63.39 & 51.58 & \multirow{3}{*}{$\begin{array}{c}13.641 \\
.009\end{array}$} \\
\hline & Media & 3.84 & 12.06 & 12.38 & 22.31 & 6.61 & \\
\hline & D.T. & 13.86 & 21.93 & 29.75 & 29.23 & 9.04 & \\
\hline \multirow{3}{*}{$\begin{array}{c}\text { Trabajo } \\
\text { en el } \\
\text { extranjero }\end{array}$} & $\begin{array}{c}\text { Rango } \\
\text { promedio }\end{array}$ & 45.00 & 46.50 & 45.00 & 59.41 & 52.69 & \multirow{3}{*}{$\begin{array}{c}14.174 \\
.007\end{array}$} \\
\hline & Media & .00 & .10 & .00 & 3.50 & .77 & \\
\hline & D.T. & .000 & .548 & .000 & 8.355 & 1.878 & \\
\hline
\end{tabular}

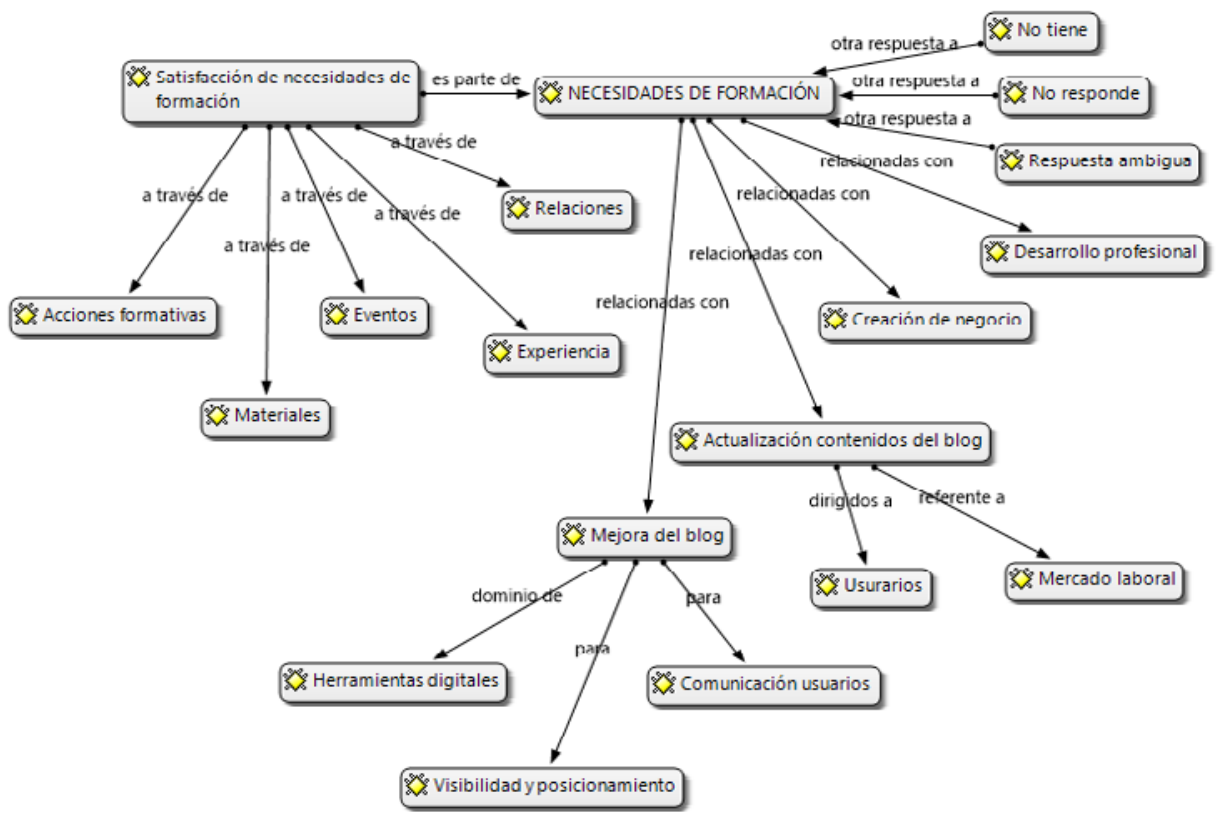

Figura 1. Necesidades formativas de los autores de los blogs 
De forma cualitativa, con la intención de cumplir con el tercer objetivo, en la figura 1 se establecen las necesidades formativas de los 65 blogueros que deciden participar en la segunda etapa de esta investigación, a partir de la respuesta a esta cuestión específica en la entrevista llevada a cabo. La figura 1 es el resultado de la reducción de los datos textuales a una red de contenidos o significados interrelacionados (network).

Las necesidades de formación expresadas se relacionan, en primer lugar, con la mejora del propio blog $\{36-4\}$. Las respuestas dadas se dividen en:

- Adquirir un dominio mayor de diferentes herramientas digitales $\{21-1\}$ que permitan la programación, diseño, desarrollo y gestión de páginas web (PHP, CMS); un mejor manejo del propio blog a partir de distintos softwares de código abierto (Wordpress, Blogger), y la implementación de recursos propios (vídeos, materiales interactivos).

- Mejorar la visibilidad y el posicionamiento del blog en Internet $\{23-1\}$ a través de una mayor difusión. Para ello consideran básicos contenidos relacionados con el marketing digital (SEO, SEM), la gestión de redes sociales desde una perspectiva profesional y la creación de una marca personal.

- Posibilitar una relación y comunicación más fluida y directa con los lectores del blog $\{5-1\}$, para lo que formarse en técnicas como Storytelling o Copywriting se convierte en fundamental.

En segundo lugar, las necesidades manifestadas por los autores de los blogs también se relacionan con la actualización permanente de los contenidos de los mismos $\{20-3\}$, que se dividen en:

- Contenidos dirigidos a los usuarios $\{20-1\}$ para hacer posible su desarrollo personal y profesional. Es por ello que les interesa profundizar en aspectos del proceso orientador, así como estar al día en nuevas tendencias dentro del ámbito de los recursos humanos. Resaltan temáticas como marca personal, el proceso de búsqueda activa de empleo, las redes sociales enfocadas a la consecución de un trabajo, el proceso de toma de decisiones o la efectividad/productividad personal.

- Contenidos referentes al mercado laboral $\{4-1\}$, en cuanto a estar al día en tendencias empresariales, crecimiento de distintos sectores, perfiles profesionales más demandados, problemáticas de determinados colectivos o problemas sociales.

En tercer lugar, los participantes manifiestan necesidades formativas en cuanto a la creación de negocio, pero con una frecuencia de respuesta mucho menor que las anteriores \{3-1\}. Esta necesidad se relaciona con la dirección, gestión y desarrollo de una empresa.

Por último, también con una frecuencia de respuesta baja $\{3-1\}$, se encuentra la necesidad de formación para el propio desarrollo profesional, adquiriendo para ello distintas competencias transversales, como puede ser la digital o la competencia idiomática.

Otras respuestas dadas a la cuestión de las necesidades formativas son que no tienen $\{7-1\}$, no responden $\{1-1\}$, en blanco $\{1-1\}$ o una contestación ambigua en la que no se concreta ninguna necesidad, más bien se defiende o reivindica la importancia de la formación continua $\{5-1\}$. 
La formulación de preguntas abiertas permite obtener una mayor riqueza en las respuestas y prueba de ello es que algunos de los autores de los blogs han complementado sus necesidades formativas con la manera en la que las satisfacen: acciones formativas $\{5-1\}$, consulta y lectura de distintos materiales (informes, estadísticas, trabajos de investigación, manuales, páginas web, otros blogs, tutoriales, etc.) de forma autodidacta $\{20-1\}$, asistencia a eventos como congresos $\{1-1\}$, a través de su propia experiencia laboral $\{2-1\}$ y las relaciones profesionales establecidas $\{2-1\}$.

\section{Discusión y conclusiones}

La blogosfera se ha convertido en un nuevo espacio, virtual e improvisado, para el desarrollo de la orientación profesional. Las posibilidades de Internet en orientación son numerosas y pueden aprovecharse a partir de la utilización de los blogs como recurso y medio de interacción con los usuarios en procesos de e-orientación; pero podría obtenerse un beneficio mayor si se conocen en profundidad las prácticas puestas en marcha a través de este nuevo contexto virtual de intervención orientadora. Ante ello se convierte en prioritario conocer a los responsables de gestionar los blogs relacionados con la orientación profesional que han ido proliferando en la blogosfera.

A partir de los resultados expuestos se concluye que la formación inicial de los blogueros-orientadores es muy variada, como corroboran los trabajos de Sánchez, Álvarez, Manzano y Pérez-González (2009), Ceinos (2008), Ferrer-Sama (2009) o Velaz de Medrano (2008). En estos trabajos también se pone de manifiesto que sobresalen las titulaciones relacionadas con la educación, como Pedagogía y Psicología, por la correspondencia del perfil profesional de estos estudios con la orientación, sobre todo, en el contexto más tradicional, el educativo, aunque hoy en día debemos concebir la orientación presente en cualquier contexto y a lo largo de la vida de la persona.

En cuanto a la formación complementaria destaca la relacionada con los recursos humanos, por tratarse de una formación en la que los procesos orientadores tienen cada vez mayor cabida y consideración. La nueva visión del personal de las organizaciones, como verdaderos activos de valor capaces de marcar la diferencia, hace que dentro de la gestión estratégica de recursos humanos se contemplen funciones que promueven el desarrollo de la carrera profesional, para poder cumplir así el objetivo de retener a los empleados más deseables (Dolan, Valle y López, 2014).

Otra opción formativa es la de coaching, en auge en los últimos tiempos, aunque escasamente formalizada, pero también relacionada con la orientación. Álvarez y Bisquerra (2012) la definen como una técnica de orientación que se ha ido abriendo camino en la empresa. Consiste en promover la definición de metas y objetivos, así como el entrenamiento en competencias tanto personales como profesionales. Tal vez por este vínculo tan estrecho entre coaching y orientación, las personas con titulaciones afines a la educación no suelen considerar esta opción, por su formación universitaria de base (Bisquerra, 2008), como demuestran los resultados de este trabajo.

En ambas opciones (RR.HH. y Coaching) son los participantes sin estudios universitarios y ADE los que menos escogen estas opciones, ¿será su experiencia profesional la que les lleve a publicar contenidos relacionados con la orientación profesional? 
Atendiendo a este aspecto, la ocupación más común de los blogueros es la de orientador laboral, seguida de la de consultor o técnico en RR.HH, hecho que parece lógico, dada la temática principal de los blogs. En cuanto al sexo, como demuestran otros estudios, existe una predominancia de los hombres frente a las mujeres en la blogosfera del contexto hispanohablante (Garrido y Lara, 2008; Merchan, García e Iturregui, 2013), siendo el grupo de orientadores donde las mujeres tienen una mayor presencia. Este grupo es el que presenta también una frecuencia de publicación más alta, en torno a 20 post mensuales. Esto puede ser debido a la concienciación de la importancia de su labor en la sociedad actual, aprovechando una herramienta 2.0 para llegar a un mayor número de personas.

En relación a los contenidos, las temáticas más publicadas se centran en ofertas de empleo privado, actualidad del mercado laboral, competencias transversales, ofertas de empleo público y búsqueda activa de empleo. Algunas de ellas parecen responder a la necesidad apremiante de encontrar trabajo (ofertas y búsqueda de empleo), pero las restantes (mercado laboral y competencias transversales) pueden aportar una perspectiva más amplia de cara al aumento de la empleabilidad y al desarrollo profesional.

Estos blogs dan a conocer documentos, enlaces de interés, actividades, programas, iniciativas, materiales de apoyo, etc., dirigidos a los usuarios, pero también sirven para la interacción entre orientadores. De ahí que los blogs sean recursos ideales tanto para crear comunidades virtuales, como para liberar al orientador de funciones como la de informar, disponiendo así de más tiempo para el desarrollo de otras en las que la relación directa se hace imprescindible- asesoramiento, consulta, formación- (Ceinos, 2008, 2009).

En cambio, la categoría con menos publicaciones hace referencia al orientador profesional, lo que indica que la blogosfera, de momento, no es utilizada como espacio de intercambio entre profesionales y aún no se han creado verdaderas comunidades virtuales que trabajen en pos de la profesionalización del orientador.

Igualmente, en cuanto a la relación de los contenidos publicados en los blogs con otras variables, se aprecian diferencias significativas cuando se habla de la tipología del mismo. En este caso, los blogs corporativos muestran un promedio mayor de publicación en nueve categorías (Tabla 5). Esto puede ser debido al carácter de negocio de éstos y, en consecuencia, a la búsqueda de una mayor visibilidad en la Red. Como se pone de manifiesto en otro trabajo (Martínez, Pérez, González, González y Martínez, 2017), las diferencias significativas en esas categorías específicas también pueden responder a una concepción más práctica de la orientación por parte de los blogs corporativos, intentando proporcionar una respuesta más inmediata a los usuarios.

Las entradas realizadas según su formación inicial presentan diferencias significativas únicamente en la categoría de competencias transversales, siendo los grupos sin estudios universitarios, titulaciones técnicas y ADE quienes más publican sobre esta temática, ¿podrían considerar este contenido de mayor importancia dada su experiencia de reconversión profesional?

En lo relativo a la formación complementaria, se encuentran únicamente diferencias significativas en la publicación de contenidos relativos a coaching, siendo los blogueros que han optado por esta formación los que publican más post al respecto, lo que parece lógico, sobre todo, para promocionarse profesionalmente en algunos círculos en los que el coaching es aún novedoso. 
En cuanto a las publicaciones según la ocupación de los participantes son los orientadores laborales los que más postean de ofertas de empleo (privadas y públicas), búsqueda activa de empleo y trabajo en el extranjero, tal vez porque estas sean las demandas que atienden con mayor frecuencia en los distintos servicios de orientación en los que trabajan, haciéndolo así extensible al entorno virtual con la intención de satisfacer un mayor número de necesidades.

Por último, a partir de la percepción de los propios participantes, se establecen como necesidades formativas aquellas cuya satisfacción permite una mejora del blog, ya que implican el manejo de diferentes herramientas, técnicas y contenidos que no sólo ayudarán en un mejor diseño, desarrollo y gestión del mismo, sino que, además, contribuirán a lograr una mejor posición en la blogosfera; en este sentido, Echeverría Ezponda (2001, p. 289) afirma que "el espacio electrónico exige habilidades y destrezas específicas para intervenir activamente en él".

Los blogueros también manifiestan como necesidad permanecer actualizados en diferentes contenidos relacionados con el proceso orientador, sobre todo, los que implican nuevas tendencias, para favorecer al máximo el desarrollo personal y profesional de los usuarios. Orihuela (2004) resalta, como una de las características más notables de los blogs, su actualización y conexión con los lectores, ya que "con sus herramientas y su cultura, los bloggers están contribuyendo a hacer realidad el sueño de los visionarios de la Red: generar un vasto repertorio universal navegable, personalizable y actualizado de información transformada en conocimiento" (p. 40). ¿Será posible que ese repertorio se haga realidad para la orientación profesional con la conformación de comunidades virtuales de práctica que beneficien tanto a usuarios como a profesionales?

Son numerosos los interrogantes que se derivan de este trabajo y en los que se pretende profundizar y avanzar desde esta línea de investigación recién iniciada. A la luz de los primeros resultados, la intención es realizar entrevistas en profundidad con los blogueros e incidir en aspectos no considerados hasta el momento.

Poner en relación la realidad on y off line de la orientación profesional es un reto importante y necesario. Esto implica colaboración, coordinación, integración y coherencia en pos de una mejora de la calidad de los servicios de orientación, independientemente de que estos sean de carácter presencial o virtual. Trabajar a partir de la consecución de objetivos comunes genera líneas de actuación conjunta, proyectos colaborativos y un largo etcétera de compromisos e intervenciones surgidas a partir de la creación de una comunidad que pueda repercutir en el avance de la profesionalización de la orientación. En todo este proceso, las herramientas 2.0 tienen mucho que aportar, por lo que los primeros pasos se dirigen a dilucidar los contenidos y prácticas que se llevan a cabo en la blogosfera de la e-orientación profesional, así como a conocer, describir y perfilar a los profesionales que las realizan, puesto que la calidad de la intervención está en sus manos.

\section{Referencias}

Álvarez, M., \& Bisquerra, R. (2012). Orientación educativa. Modelos, áreas, estrategias y recursos. Madrid: Wolters Kluwer España. 
Aznar, V., \& Soto, J. (2010). Análisis de las aportaciones de los blogs educativos al logro de la competencia digital. Revista de Investigación en Educación, 7, 83-90.

Barraza, A. (2013). Presencia discursiva del estrés académico en la blogósfera. Un análisis de contenido de treinta blogs. Revista Iberoamericana de Psicología: Ciencia y Tecnología, 6(2), 45-54.

Bisquerra, R. (2008). Coaching: un reto para los orientadores. Revista Española de Orientación y Psicopedagogía, 19(2), 163-170.

Blood, R. (2000). Weblogs: a history and perspective. At the Interface: New Intimacies, New Cultures, 5. Recuperado de https://ejpost.files.wordpress.com/2010/08/ weblogsa-history-and-perspective.pdf

Bordignom, F.R.A. (agosto, 2007). Hacía una caracterización del blogger argentino. Trabajo presentado en el II Congreso Internacional de Educación a Distancia y Tecnologías Educativas, Buenos Aires (Argentina).

Ceinos, C. (2008). Diagnóstico de las competencias de los orientadores laborales en el uso de las Tecnologías de la Información y de la Comunicación. (Tesis Doctoral). Recuperado de https://minerva.usc.es/xmlui/handle/10347/2486

Ceinos, C. (2009). Las Tecnologías de la Información y Comunicación (TIC) en Orientación Profesional. En L. Sobrado \& A. Cortés (Coords.), Orientación Profesional: nuevos escenarios y perspectivas (pp. 183-201). Madrid: Biblioteca Nueva.

Comisión Europea (2013). Comprender las políticas de la Unión Europea Europa 2020: la estrategia europea de crecimiento. Luxemburgo: Oficina de Publicaciones de la Unión Europea.

Cuevas, V.J. (2011). El blog como herramienta de orientación académica. En J. Martín y A. Cobos (Coord.), V Encuentro Nacional de Orientación: Educar y orientar en la diversidad (pp. 299-308). Sevilla: COPOE.

Dolan, S.L., Valle, R., \& López, A. (2014). La gestión de personas y del talento. La gestión de los recursos humanos en el siglo XXI. Madrid: McGraw Hill.

Echeverría Ezponda, J. (2001). Educación y Sociedad de la Información. Revista de Investigación Educativa, 19(2), 277-289.

Estalella, A. (2005). Anatomía de los blogs. La jerarquía de los visible. Revista Telos, 65. Recuperado de https://telos.fundaciontelefonica.com/telos/articulocuaderno. asp@ idarticulo $=9 \& \mathrm{rev}=65 . \mathrm{htm}$

Fernández González, M. (2015). Orientación profesional para el empleo en la web 2.0 y preparándonos para la 3.0. Badajoz: Comisiones Obreras de Extremadura.

Ferrer-Sama, P. (2009). La acreditación de los profesionales de la orientación. En L.M. Sobrado \& A. Cortés (Coords.), Orientación Profesional. Nuevos escenarios y perspectivas (pp. 341-356). Madrid: Biblioteca Nueva.

Ferreres, G., \& Garrido, F. (2006). El perfil del blogger español. En J.M. Cerezo (Dir.), La blogosfera hispana: pioneros de la cultura digital (pp.52-70). Madrid: Fundació France Telecom España.

Flores, D. (2008). En busca del sujeto extraviado. Reflexiones en torno al estudio de blogs. Diálogos de la Comunicación, 76. Recuperado de http://www.dialogosfelafacs. net/76/articulos/ pdf/76DorismildaFlores.pdf 
Fumero, A. (2005). Un tutorial sobre blogs. El abecé del universo blog. Revista Telos, 65. Recuperado de https://telos.fundaciontelefonica.com/telos/articulo cuaderno.asp@ idarticulo $=1 \&$ rev $=65 . \mathrm{htm}$

Garrido, F., \& Lara, T. (2008). Perfil del blogger hispano. III Encuesta a Bloggers. Diálogos de la Comunicación, 76. Recuperado de http://dialogosfelafacs. net/76/articulos/ pdf/76GarridoLara.pdf

Hernández, R., Fernández-Collado, C., \& Baptista, P. (2007). Metodología de investigación (4⿳亠丷厂 ed.). México: McGraw Hill.

Huertas, A. (2010). Blogging PR: análisis de contenidos de los blogs de relaciones públicas. En M.C. Carretón (Coord.), Las relaciones públicas en la sociedad del conocimiento (pp. 362-380). Alicante: Asociación de Investigadores en Relaciones Públicas.

Instituto Nacional de Estadística [INE]. (2016). Encuesta sobre Equipamiento y Uso de Tecnologías de Información y Comunicación en los Hogares. Año 2016. Madrid: Autor.

Jou, B. (2009). Blogs ¿Para qué? marcoELE-Revista de Didáctica Español Lengua Extrajera, 8, 1-12. Recuperado de http://marcoele.com/blogs-para-que/

Martínez, M., Pérez, J., González, C., González, N., \& Martínez, P. (junio, 2017). La orientación profesional en la blogosfera. Trabajo presentado al XVIII Congreso Internacional de Investigación Educativa, Salamanca (España).

Merchán, I., García, D., \& Iturregui, L. (2013). Las chicas no son blogueras. Temática y estructura empresarial de los principañes blogs españoles desde una perspectiva de género. Revista Telos, 94. Recuperado de https://telos.fundaciontelefonica.com/ seccion $=1268 \&$ idioma $=$ es_ES\&id=2013021317590003\&activo=6.do

Muñoz-Carril, P.C., \& González-Sanmamed, M. (2014). Posibilidades de la web 2.0 en orientación educativa: un estudio exploratorio sobre su presencia en las web de los departamentos de orientación de secundaria. Revista Española de Orientación y Psicopedagogía, 25(3), 36-55.

Orihuela, J.L. (2004). Los weblogs: revolución y consolidación. Revista Latinoamericana de Comunicación CHASQUI, 85, 36-41.

Orihuela, J.L. (2006). La revolución de los blogs. Madrid: La Esfera de los Libros.

Pantoja, A., \& Zwierewicz, M. (2008). Procesos de orientación en entornos virtuales de aprendizaje. Revista Española de Orientación y Psicopedagogía, 19(3), 282-290.

Romero, C., \& Montilla, M. (2015). La utilización de las TIC en la orientación educativa: un estudio exploratorio sobre la situación actual de uso y formación entre los profesionales de la orientación. Revista Española de Orientación y Psicopedagogía, 26(3), 78-95.

Sánchez, M.F., Álvarez, B., Manzano, N., Pérez-González, J.C. (2009). Análisis de las competencias del orientador profesional: implicaciones para su formación. Revista Española de Orientación y Psicopedagogía, 20(3), 284-299.

Sáez Vacas, F. (2005). La blogosfera: Un vigoroso subespacio de comunicación en Internet. Revista Telos, 64. Recuperado de https://telos.fundaciontelefonica.com/ telos/articulotribuna.asp@idarticulo=3\&rev=64.htm

Santana, L.E. (2013). Orientación profesional. Madrid: Síntesis.

Santana, L.E., \& Santana, A. (2011). Las TIC: una herramienta para la orientación educativa y profesional de los jóvenes. En C.S. González González (Dir.), Nuevas tendencias en TIC y Educación (Vol. 1, pp. 57-69). Madrid: Budok. 
Sobrado, L.M., \& Ceinos, C. (2011). Tecnologías de la Información y de la Comunicación. Uso en orientación profesional y formación. Madrid: Biblioteca Nueva.

Sobrado, L.M., Ceinos, C., \& García, R. (2012). Utilización de las TIC en orientación profesional: experiencias innovadoras. Revista Mexicana de Orientación Educativa, 9(23), 2-10.

Velaz de Medrano, C. (2008). Formación y profesionalización de los orientadores desde el enfoque de competencias. Educación XX1, 11, 155-181.

Work Economic Fourm [WEF]. (2016). The future of jobs employment, skills and workforce strategy for the Fourth Industrial Revolution. Recuperado de http://www3.weforum. org/docs/WEF_Future_of_Jobs.pdf

Fecha de recepción: 11 de octubre de 2017.

Fecha de revisión: 18 de octubre de 2017.

Fecha de aceptación: 18 de octubre de 2017. 